A C T A C H E I OA SCANDINAVIOA 1 (1947) $566-570$

\title{
Neue Versuche mit beweglicher calorimetrischer Bombe
}

\author{
LENNART SMITH Und LARS BJELLERUP
}

Organische Abteilung, Chemisches Institut der Universität, Lund, Schweden

Tn der Svedberg-Festschrift berichteten Smith und Sunner über Bestimmun-
gen von Verbrennungswärmen mit schaukelnder, bzw, rotierender Bombe. Es wurde gezeigt, dass die Verbrennungswärmen von chlorhaltigen Substanzen durch Schaukeln der Bombe und von bromhaltigen durch Rotieren derselben mit genügender Genauigkeit bestimmt werden konnten.

Die Schaukelversuche schienen indessen insofern nicht zufriedenstellend, als die benutzte, ziemlich komplizierte mechanische Vorrichtung nur eine verhältnismässig kleine Schaukelamplitude gestattete: nur etwa $50^{\circ}$ von der vertikalen Stellung. Infolgedessen war die Reduktion des Chlors durch die arsenige Säure der Bombenlösung, wahrscheinlich eben wegen unzureichender Bewegung der Bombe, bei sehr chlorreichen Substanzen unvollständig, wenn auch der Fehler in den Verbrennungswärmen zu vernachlässigen war. Daneben war die Korrektur für die Friktionswärme bei dieser Vorrichtung nicht unbedeutend: $0,001^{\circ}$ bei einer Temperaturerhöhung von etwa $1^{\circ}$.

Die Erfinder der ersten Schaukelbombe (Popoff und Schirokich ${ }^{\mathbf{1}}$ ) erwähnen, dass ihre Bombe mit Hilfe von zwei Schnüren bewegt wurde, die an einem auf der Bombenachse befindlichem Rad befestigt waren. Weitere Einzelkeiten werden nicht angegeben.

Nun schien es zunächst möglich, mit Hilfe von Rad und Schnüren die vertikal aufgehängte Bombe rotieren zu lassen, wodurch ja eine ebenso vollkommene Berührung von Gas- und Flüssigkeitsphase erzielt werden könnte wie durch Schaukeln. - Die in der Mitteilung von Smith und Sunner erwähnte rotierende Bombe beschreibt eine Bewegung in horisontaler Lage. Wir hatten ursprünglich beabsichtigt, die schaukelnde Bewegung einfach durch Handkraft unter Zuhilfenahme eines Metronoms zu bewirken. Durch Wegnahme der komplizierten Schaukelvorrichtung von Smith und Sunner könnten auch viel grössere Amplituden erreicht werden. Da man erwarten konnte, dass eine Bewegung durch Handkraft nicht so gut reproduzierbar 
Fig. 1. Die zu den Versuchen benutzte Schaukelvorrichtung.

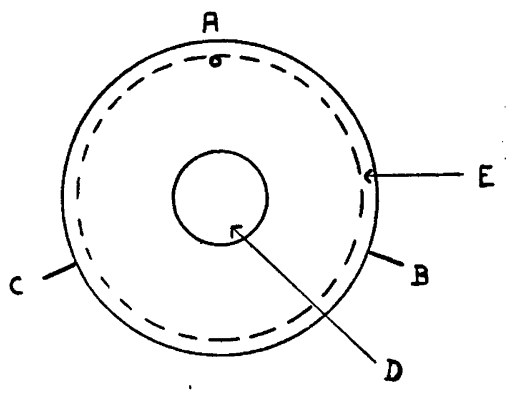

wäre wie die durch eine mechanische Vorrichtung bewirkte, haben wir zu den Versuchen mit Schaukelbewegung die in Fig. 1 wiedergegebenen Konstruktion* benutzt:

In der Figur stellt $\mathbf{E}$ das auf der Aufhängungsachse (D) befestigte Rad dar, B und C sind Zapfen am Rade. Bei A hat ein Kupferdraht (Diameter $0,3 \mathrm{~mm}$ ) seinen Endpunkt. Von A aus geht der Draht in einer Spur bis B, wendet um den Zapfen und geht über $\mathrm{A}$ weiter nach $\mathrm{C}$, nach Wendung weiter nach B, u. s. w. Beim Aufwinden des Drahtes (durch ein Loch in Kalorimeterdeckel), welches mit konstanter Geschwindigkeit geschieht, erhält die Bombe eine schaukelnde Bewegung, und zwar können Amplituden bis $100^{\circ}$ in jeder Richtung erzielt werden. Betreffs möglicher Wasserverluste durch den Draht und Friktionswärme gilt, was Smith und Sunner hinsichtlich der horizontalen Rotationsbombe angeführt haben. Besondere Bestimmungen dieser Grössen brauchen nicht vorgenommen zu werden.

Man kann nach Belieben Verbrennungen entweder mit schaukelnder oder mit rotierender Bombe ausführen. Das letztgenannte Verfahren ist aus dem Grunde am vorteilhaftesten, da bei vollständiger Umdrehung die Reduktion des freien Halogens am schnellsten vor sich geht und alle Konzentrationsunterschiede sicher ausgeglichen werden. Indessen hat es sich herausgestellt, dass beim Rotieren Tiegel und Schild bisweilen in der festen Armatur der Bombe hängen bleiben, was ein Misslingen der Verbrennung zur Folge haben kann. Am vorteilhaftesten scheint es zu sein, in einem Versuche die Bombe zuerst sohaukeln, dann rotieren zu lassen (s. u. bei Äthylenbromid).

Die Methode wurde mit Hilfe von Bromverbindungen ausgearbeitet, die ja in höherem Grade als Chlorverbindungen geeignet sind, zur Prüfung auf die Verwendbarkeit der Methode zu dienen. Es wurden $\alpha$-Bromnaphthalin, Brombenzol und Äthylenbromid verbrannt.

* Konstruktion von Bjellerup. 
Bei Verbrennung von a-Bromnaphthalin führte die Bombe 20 halbe Schaukelbewegungen (Zeit $75 \pm 3$ s) aus, bei Brombenzol 12 vollständige Umdrehungen (Zeit $82 \pm 4 \mathrm{~s}$ ) und schliesslich bei Äthylenbromid 6 halbe Schaukelbewegungen nebst 9 Umdrehungen (Zeit $86 \pm 4 \mathrm{~s}$ ). Die Bewegungen wurden $30 \mathrm{~s}$ nach der Zündung in Gang gesetzt. Zu dieser Zeit ist die Verbrennung der Substanz vollständig.

Die Vollständigkeit der Bromreduktion wurde zweimal in der Weise geprüft, dass unmittelbar nach dem Abschlusse einer Verbrennung die Bombengase durch Natronlauge in einer Effektivwaschflasche geleitet wurden. Es konnte in der Lauge kein Bromid nachgewiesen werden. Die Armatur ist beim Öffnen der Bombe oft von einer braungefärbten Flüssigkeit befeuchtet (wahrscheinlich freies Brom in Bromid gelöst). Wir fanden indessen, dass die von diesem Fehler herrührende Korrektur sehr klein ist. Auch wenn angenommen wird, dass alles Brom (auch das Bromid) in der Armaturflüssigkeit als freies Element vorliegt, betrüge die Korrektur nur etwa $-0,4$ cal.

Die Substanzen (alle drei flüssig) wurden nach Verkade und Coops ${ }^{2,3}$ zur Verbrennung in kleinen Glaskölbchen zusammen mit Paraffin eingewägt. Einige Einzelheiten wurden verbessert zur Vermeidung von zufälliger Russbildung, wie später von Bjellerup beschrieben wird. Übrige experimentelle Anordnungen und Versuchsbedingungen nach Smith und Sunner, deren Präparate auch benutzt wurden: sie wurden durch Destillation gereinigt und ihre Reinheit durch Bestimmung von Dichte und Brechung kontrolliert. Wir finden es überflüssig, diesbezügliche Ziffern hier anzuführen. Die gefundenen Konstanten stimmten gut mit den Angaben der Literatur überein.

Für die wahrscheinlich sehr kleine Friktionswärme wurde in der Weise korrigiert, dass die Bestimmung des Wasserwertes (mit Benzoesäure) in genau derselben Weise wie die Bestimmung der Verbrennungswärme geschah, für $\alpha$-Bromnaphthalin also mit Schaukelbewegung, für Brombenzol mit Umdrehen. Da zwischen diesen beiden Bestimmungen einige Änderungen in der Bombe vorgenommen wurden, die den Wasserwert veränderten, können wir auf Grund des vorliegenden Ziffermaterials nicht behaupten, dass der "Schaukelwasserwert" und der "Umdrehungswasserwert" gleich gross sind. Dass dies der Fall ist, halten wir für sehr wahrscheinlich. Eine Bestimmung unter Schaukeln + Umdrehen ergab den Wasserwert 4709 cal, während bei Umdrehen $4710 \pm 2$ cal getunden wurde. Für Äthylenbromid (verbrannt mit Schaukeln + Umdrehen) wurde mit dem Schaukel-Wasserwert gerechnet.

Als Beispiel für die Genauigkeit einer Wasserwertbestimmung (Umdrehen) führen wir an (Benzoesäure 0,9 g, Temp.-Erhöh. 1,1-1,2 ${ }^{\circ}$ ):

$\begin{array}{llllll}4692 & 4694 & 4697 & 4694 & 4692 & M=4694 \pm 1,5 \text { cal }\end{array}$


Beim Schaukeln ist die Genauigkeit mindestens ebenso gut. Verbrennungswärme des Paraffinöls $10990 \pm 4$ cal; Bombenlösung 30,00 ml etwa $0,25 N$ arsenige Säure; Sauerstoffdruck $30 \mathrm{Atm}$.

In den Tabellen bedeuten: $m=m g$ Substanz; $m_{1}=m g$ Paraffinöl; $Q=$ Verbrennungswert in cal/g; red. = reduziertes Brom in \%; $\mathrm{Br}=$ wiedergefundenes $\mathrm{Brom}$ in $\%$.

Tabelle 1. $\alpha$-Bromnaphthalin.

$\begin{array}{ccccc}m & m_{1} & Q & \text { red. } & \mathbf{B r} \\ \mathbf{4 4 1 , 1 0} & 296,80 & 5800 & 89 & \mathbf{9 8 , 6} \\ \mathbf{4 3 7}, 90 & 298,90 & 5801 & 90 & \mathbf{9 9 , 8} \\ \mathbf{4 4 0 , 6 0} & \mathbf{3 0 1 , 8 0} & 5802 & \mathbf{9 1} & \mathbf{9 9 , 2} \\ \mathbf{4 5 7 , 5 5} & \mathbf{2 9 4 , 4 0} & 5798 & \mathbf{9 1} & \mathbf{9 9 , 4} \\ & \mathrm{M}=\mathbf{5 8 0 0} \pm 1 & \text { (Smith und Sunner: } & \mathbf{5 8 0 0 , 5} \pm \mathbf{1 , 5} \text { ) }\end{array}$

Tabelle 2. Brombenzol.

$\begin{array}{cccc}m & m_{1} & Q & \text { red. } \\ 491,30 & 294,90 & 4737 & 91 \\ 455,00 & 224,30 & 4738 & 92 \\ 466,95 & 315,05 & 4736 & 90 \\ & \text { M }=4737 \text { (Smith und Sunner: } 4743 \pm 1 \text { ) }\end{array}$

Tabelle 3. Äthylenbromid.

\begin{tabular}{|c|c|c|c|}
\hline$m$ & $m_{1}$ & $Q$ & red. \\
\hline 473,00 & 431,00 & 1576 & 94 \\
\hline 537,85 & 432,30 & 1578 & 95 \\
\hline 423,00 & 460,20 & 1580 & 92 \\
\hline
\end{tabular}

Einige Werte, die unter Russbildung verliefen, wurden verworfen. Die Abweichung bei Äthylenbromid liegt noch innerhalb der Versuchsfehler, kann andererseits auch davon herrühren, dass dieses Präparat nicht so rein gewesen ist wie dasjenige von Smith und Sunner.

Washburn-Korrektur ist nicht angebracht worden.

\section{ZUSAMMENFASSUNG}

Die Untersuchung zeigt, dass die hier benutzte vereinfachte Methode für Schaukeln und Umdrehen einer calorimetrischen Bombe für die Bestimmung der Verbrennungswärmen von bromhaltigen (und auch von chlorhaltigen) Verbindungen gut verwendbar ist. 
Für Unterstützung von Seiten des Kemiska Nobelfonden spricht der eine von uns (Sm.) seinen herzlichen Dank aus.

\section{LITERATUR}

1. Popoff, M. M., und Schirokich, P. K. Z. physik. Chem. A 167 (1933) 183.

2. Verkade, P.E., und Coops, J. Rec. trav. chim. 45 (1926) 545.

3. Eftring, E. Diss. Lund (1938).

Eingegangen am 8. Juni 1947. 